\title{
On the path to improved muscle functional testing in cancer patients
}

\author{
Daria Neyroud $^{1}$ \\ Published online: 11 June 2020 \\ (c) Springer-Verlag GmbH Germany, part of Springer Nature 2020
}

Patients diagnosed with cancer often present with compromised muscle health. For instance, up to $80 \%$ of cancer patients experience involuntary and ongoing loss of skeletal muscle mass that may or may not associate with concomitant loss of fat mass (Fearon et al. 2011). This cancer-associated loss of skeletal muscle mass, known as cancer cachexia, associates with muscle weakness and reduced functional capacity, which further contribute to the deterioration of quality of life and reduced ability to independently perform common daily tasks in cancer patients. In addition, the presence of cachexia limits cancer patients' eligibility for cancer treatments as reductions in skeletal muscle mass associate with increased treatment toxicity and reduced treatment efficacy. Noteworthy for eligible cancer patients, cancer treatments may further precipitate skeletal muscle loss and dysfunction. Whether it is caused by cancer itself or cancer treatments, skeletal muscle loss and/or dysfunction results in cancer-related fatigue defined as the perception of unusual tiredness negatively impacting cancer patients' functioning, increases the risk of developing severe secondary comorbidities such as cardiovascular diseases and reduces survival.

Despite extensive research in the field, cancer-associated skeletal muscle loss and dysfunction remain incompletely understood and currently no approved treatment exists to counter this multifactorial pathology. The current failure to develop treatments relies in part in the often late diagnosis caused by a poor general awareness of this condition and lack of diagnostic tools. For instance, we currently lack methods to evaluate and monitor muscle function that are sensitive enough to detect alterations relevant for daily physical functioning in this patient population (Laird et al. 2018). Since the success of clinical trials for cachexia is dependent on improvement of muscle function, it is primordial to develop, validate, and implement such methods.

Daria Neyroud

daria.neyroud@ufl.edu

1 Department of Physical Therapy, University of Florida, Gainesville, FL 32610, USA
Currently, physical function, muscle weakness, and cancer-related fatigue are commonly evaluated as patientreported symptoms by the mean of questionnaires, whereas only in rare occasions, more objective methods such as the six-minute walk test or handgrip forces are being used. Given that muscle performance might be affected by several processes resulting from the interaction of intramuscular, nervous, systemic, and psychological factors, it is of importance to develop evaluation methods able to assess the contribution of these different factors.

In this context, the methodology used in the study conducted by Lavigne et al. (2020) is of particular interest. This study examined the effects of radiotherapy with or without concomitant chemotherapy on neuromuscular function and fatigability (i.e. muscle capacity to perform some work) of the knee extensors of patients diagnosed with head and neck malignancies. The authors took advantages of gold-standard methods that are routinely used to assess neuromuscular function combined with common patient-reported outcomes. In doing so, Lavigne et al. demonstrated that voluntary and electrically induced force measurements could be conducted in cancer patients and that those measurements were sensitive enough to detect treatment-induced deterioration in muscle function. They further showed that patient-reported increased perception of fatigue and reduced health-related quality of life correlated with the reduced exercise capacity observed after treatment, whereas no correlation between these patient-reported outcomes and muscle cross-sectional area (i.e. an index of muscle mass obtained via ultrasonography) was found. Although Lavigne et al. study did not comprise a healthy control group for allowing baseline comparison and investigating the sensitivity of their methods to capture the extent and etiology of potential muscle impairments in response to cancer itself, previous studies have demonstrated that maximal voluntary force and electrically evoked force evaluations were capable of detecting elbow flexor (Cai et al. 2014; Yavuzsen et al. 2009) and knee extensor (Fukushima et al. 2019) muscle weakness in cachectic patients. 
Overall, the methodology applied by Lavigne et al. (2020) shows great promise as it appears to be sensitive enough to detect cancer treatment-induced changes in muscle function of a large muscle group involved in most daily activities. Given that this methodology would be easy to implement in a clinical setting, Lavigne et al. study opens interesting avenues to obtain greater knowledge of the extent of physical function limitations throughout the progression of cancer and cancer treatments. Future studies validating this methodology in different cancer populations are now required. Moreover, longitudinal studies in cancer patients, who are naïve to treatments as well as in cancer patients undergoing different cancer treatments, would represent important next steps. These future studies could contribute toward the implementation of this methodology for diagnosis and tracking of cancer-associated and treatment-associated skeletal muscle loss and dysfunction. Implementation of this methodology for routine measurements would provide invaluable information to guide the development of strategies to counter cancer-associated compromised muscle health and subsequent consequences.

Author contributions DN wrote this editorial.

\section{Compliance with ethical standard}

Conflict of interest The author declares no conflict of interest.

\section{References}

Cai B, Allexandre D, Rajagopalan V, Jiang Z, Siemionow V, Ranganathan VK, Davis MP, Walsh D, Dai K, Yue GH (2014) Evidence of significant central fatigue in patients with cancer-related fatigue during repetitive elbow flexions till perceived exhaustion. PLoS ONE 9(12):e115370

Fearon K, Strasser F, Anker SD, Bosaeus I, Bruera E, Fainsinger RL, Jatoi A, Loprinzi C, MacDonald N, Mantovani G, Davis M, Muscaritoli M, Ottery F, Radbruch L, Ravasco P, Walsh D, Wilcock A, Kaasa S, Baracos VE (2011) Definition and classification of cancer cachexia: an international consensus. Lancet Oncol 12(5):489-495

Fukushima T, Nakano J, Ishii S, Natsuzako A, Hirase T, Sakamoto J, Okita M (2019) Characteristics of muscle function and the effect of cachexia in patients with haematological malignancy. Eur J Cancer Care (Engl) 28(2):e12956

Laird BJA, Balstad TR, Solheim TS (2018) Endpoints in clinical trials in cancer cachexia: where to start? Curr Opin Support Palliat Care 12(4):445-452

Lavigne C, Lau H, Francis G, Culos-Reed SN, Millet GY, Twomey R (2020) Neuromuscular function and fatigability in people diagnosed with head and neck cancer before versus after treatment. Eur J Appl Physiol 120(6):1289-1304

Yavuzsen T, Davis MP, Ranganathan VK, Walsh D, Siemionow V, Kirkova J, Khoshknabi D, Lagman R, LeGrand S, Yue GH (2009) Cancer-related fatigue: central or peripheral? J Pain Symptom Manage 38(4):587-596

Publisher's Note Springer Nature remains neutral with regard to jurisdictional claims in published maps and institutional affiliations. 